\title{
Merkel Cell Carcinoma of Eyelid: A Rare Clinical Presentation and Diagnostic Challenge
}

\author{
Manpreet Singh, Swati Singh, Usha Singh", Zoramthara Zadeng \\ Department of ophthalmology, Advanced Eye Center, Post Graduate Institute of Medical Education and Research, Chandigarh, India. \\ Email: *drushasingh@gmail.com
}

Received October $27^{\text {th }}, 2012$; revised November $19^{\text {th }}, 2012$; accepted November $25^{\text {th }}, 2012$

\begin{abstract}
The purpose of this paper is to report a case of rare localised (limited to eyelid) malignant eyelid tumor in a 70 year old Indian male. Histopathology confirmed it to be a Merkel cell carcinoma. Surgical excision and eyelid reconstruction was done successfully. No local or systemic recurrence has been noted at a follow up of 2 years. The well defined nature of a highly malignant tumor, its localised presentation and management is being described in following report.
\end{abstract}

Keywords: Merkel Cell Carcinoma; Eyelid; Clinical Features; Management

\section{Introduction}

Merkel cells are cutaneous mechanoreceptors receiving touch and hair movement sensations. Merkel cell carcinoma (MCC) of eyelid is a rare aggressive tumor with less than 100 reported cases worldwide. Its predilection for elderly males, benign appearance and few typical clinical features can suggest this commonly misdiagnosed entity [1]. We hereby describe clinical presentation and management of a localized MCC of eyelid.

\section{Case Report}

A 70 year old Indian male presented to oculoplastics clinic with chief complaints of mass overright upper eyelid for last 2 months. It was painless and gradually progressive in nature. There was no history of bleeding from mass or loss of eyelashes. No history of organ transplanttation and immunosuppression. On examination, a soft to firm non tender, reddish pink mass measuring $20 \mathrm{~mm}$ (horizontally) was present on lateral half of right upper eyelid. Eyelid margin was involved and mechanical ptosis was present. Overlying skin was smooth with small to medium sized telengiectatic vessels over the surface (Figure 1). Transillumination test was positive. There was no regional lymphadenopathy. Left eye and adnexa were essentially within normal limits. Best corrected visual acuity was $20 / 60$ in both eyes attributable to nuclear sclerosis of grade 3 .

Differential diagnosis of upper eyelid squamous papilloma, acquired capillary hemangioma and Kaposi sarcoma were kept. Excisional biopsy was performed under

${ }^{*}$ Corresponding author. local anesthesia with preservation of overlying skin and eyelid margin. Mass was sent for histopathology. Report showed presence of round to oval tumor cells arranged in sheets having high nuclear: cytoplasmic ratio. Immunohistochemistry revealed positivity for CK-20, neuron specific enolase and epithelial membrane antigen, suggestive of Merkel cell carcinoma (Figures 2 and 3).

After getting the diagnosis of a malignant tumor of eyelid, patient was subjected to detailed systemic metastatic workup in the form of abdominal ultrasound, chest $\mathrm{X}$ ray and positron emission tomography. All the investigations were within normal limits. Patient was taken up for wide surgical excision (margin $5 \mathrm{~mm}$ ) and eyelid reconstruction (direct closure) within a week from primary surgery. Histopathology specimen revealed all margins, tumor free. Currently, patient is asymptomatic with no evidence of local or systemic tumor recurrence at 24 months of uneventful follow up (Figure 4).

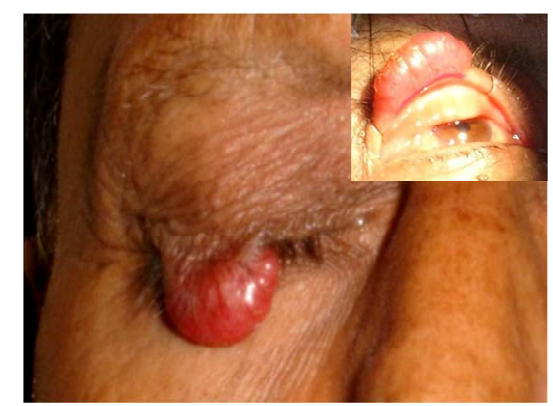

Figure 1. Photo of right eye upper eyelid (at presentation) showing a reddish-pink, well defined mass with mechanical ptosis. On eversion, mass is seen arising from eyelid margin with telengiectatic vessels over surface (inset). 


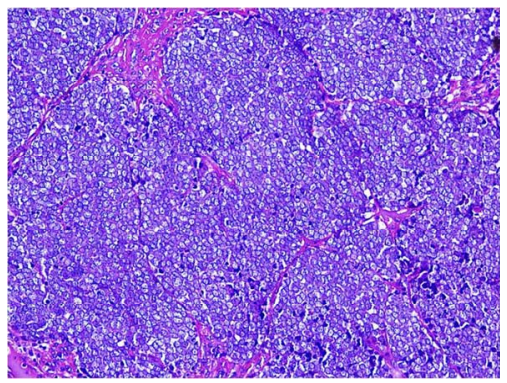

Figure 2. Histopathology shows round to oval tumor cells arranged in diffuse sheets.

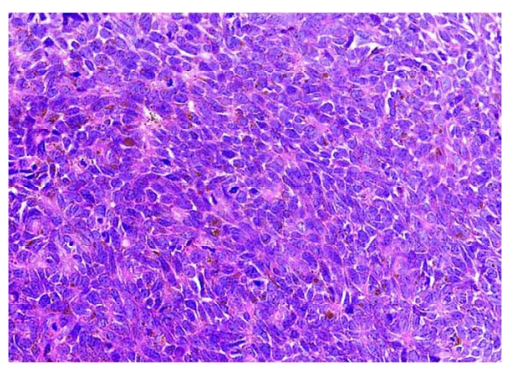

Figure 3. Immunohistochemistry shows positivity for cytokeratin 20 \& neuron specific enolase (marked with brown color).

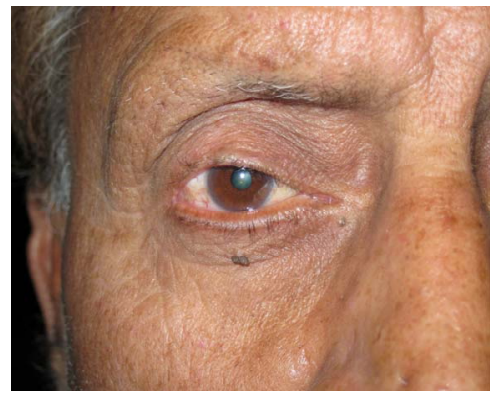

Figure 4. Post-operatively, eyelid showing normal contour with no evidence of tumor recurrence at $\mathbf{2 4}$ months.

\section{Discussion}

Upto $9 \%$ of MCC have been reported to originate from eyelids. Most commonly it involves upper eyelid arising near its margin, either sparing or causing partial eyelash loss. Its clinical benign appearance (overlying smooth skin, telangiectasia and color) can lead to delay in diagnosis and start of appropriate management, timely [2]. Risk factors include male gender, UV-B light exposure, fair skin, immunosuppression (organ transplant) [3]. Clinical diagnosis of this condition can rarely be made before histopathology.

On microscopic examination, a highly malignant tumor involving the dermis with subcutaneous extension is usually seen. It typically shows round to oval tumor cells with high nuclear: cytoplasmic ratio, scanty cytoplasm, stippled chromatin and high mitotic figures. Intervening stroma can have few congested and proliferating blood vessels. Immunohistochemistry, characterize this tumor by showing positivity for neuron specific enolase, cytokeratin and epithelial membrane antigen [4].

Differential diagnosis included Kaposi sarcoma which classically present as indolent erythematous or violaceous patch or plaques, acquired capillary hemangioma which is reddish pedunclated mass with characteristic histology showing multiple endothelial cells with abundant capillaries. Nearly $66 \%$ patients with this tumor had regional lymph node involvement at the time of diagnosis or within 18 months of presentation. Distant metastasis is seen in more than one third of patients spreading to skin, bone, brain, liver and lung [5].

The National Comprehensive Cancer Network and American Joint Committee of Cancer staging parameters have classified MCC into the following stages: stage I (primary tumor $<2 \mathrm{~cm}$ ), stage II (primary tumor $\geq 2 \mathrm{~cm}$ ), stage III (regional nodal disease), and stage IV (distant metastasis) [6]. On its basis, the 3-year survival rate of $90 \%, 70 \%, 60 \% \& 20 \%$ has been described for the above mentioned stages, respectively [7].

Surgical resection has been described as standard of care for this tumor. Wide excision $(1$ to $2 \mathrm{~cm}$ ) with tumor free margins shown by frozen section technique been advised to minimize local recurrence rate. Sentinel lymph node biopsy can be a helpful tool in determination of subclinical nodal metastases for evaluating proper extent of disease [8]. Recurrence rate of $21 \%$ has been found by a case series using wide surgical excision with the longest duration of 30 months in one case [9].

Radiotherapy and chemotherapy has been advised in management of metastatic disease, the former been more effective. Thirty eight to $68 \%$ overall 5 -year survival rate has been described for all sites [10]. However, the overall prognosis for Merkel cell tumor of eyelid is relatively poor due to its aggressive nature and potential for local recurrence. In conclusion, high clinical suspicion of Merkel cell carcinoma should be kept in a benign appearing reddish-pink eyelid mass lesion in an elderly male. Because of high malignant nature of MCC, patient should be kept on close follow up and should be investigated thoroughly for metastasis.

\section{REFERENCES}

[1] J. M. Shah, G. Sundar, K. B. Tan and Y. K. Zee, "Unusual Merkel Cell Carcinoma of the Eyelid," Orbit, Vol. 31, No. 6, 2012, pp. 425-427.

[2] S. S. Searl, J. R. Boynton, W. Markowitch and P. A. diSant'Agnese, "Malignant Merkel Cell Neoplasm of the Eyelid," Archives of Ophthalmology, Vol. 102, No. 6, 1984, pp. 907-911.

[3] R. W. Miller and C. S. Rabkin, "Merkel Cell Carcinoma and Melanoma: Etiological Similarities and Differences," Cancer Epidemiology, Biomarkers and Prevention, Vol. 8, 
No. 2, 1999, pp. 153-158.

[4] A. K. Metz, M. Jacob, U. Schmidt, K. P. Ateuhl and L. D. Leder, "Merkel Cell Carcinoma of the Eyelid: Histological and Immunohistochemical Features with Special Respect to Differential Diagnosis," Graefs Archive Clinical Experimental Ophthalmology, Vol. 236, No. 8, 1998, pp. 561-566. doi:10.1007/s004170050121

[5] A. L. Hitchcock, K. I. Bland, R. G. Laney, D. Franzini, B. Harris and E. M. Copeland 3rd, "Neuroendocrine (Merkel cell) Carcinoma of the Skin: Its Natural History, Diagnosis, and Treatment," Annals of Surgery, Vol. 207, No. 2, 1988, pp. 201-207. doi:10.1097/00000658-198802000-00015

[6] National Comprehensive Cancer Network (NCCN), "Clinical Practice Guidelines in Oncology: Merkel Cell Carcinoma," Journal of the National Comprehensive Cancer Network, Vol. 2, No. 1, 2008, pp. 80-87.

[7] M. H. Swann and J. Yoon, "Merkel Cell Carcinoma," Se- minars in Oncology, Vol. 34, No. 1, 2007, pp. 51-56. doi:10.1053/j.seminoncol.2006.10.021

[8] B. Esmaeli, A. Naderi, L. Hidaji, G. Blumenschein and V. G. Preito, "Merkel Cell Carcinoma of the Eyelid with a Positive Sentinel Node," Archives of Ophthalmology, Vol. 120, No. 5, 2002, pp. 646-648.

[9] G. B. Peters, D. R. Meyer, J. A. Shields, P. L.Causter, P. A. Rubin, T. H. Wojno, T. A. Bersani and M. Tanenbaum, "Management and Prognosis of Merkel Cell Carcinoma of the Eyelid," Ophthalmology, Vol. 108, No. 9, 2001, pp. 1575-1579. doi:10.1016/S0161-6420(01)00701-1

[10] M. Papamichail, I. Nikolaidis, N. Nikolaidis, C. Glava, I. Lentza, K. Marmagkiolis, K. Karassavsa and M. Digalakis, "Merkel Cell Carcinoma of the Upper Extremity: Case Report and an Update," World Journal of Surgical Oncology, Vol. 6, No. 7, 2008. doi:10.1186/1477-7819-6-32 